same size and, according to one theoretical model, the performance of an RFP at the reactor level could exceed that of the tokamak (J.B. Taylor, Culham).

Another approach to maintaining a stable discharge at high values of plasma current is to apply a helical field of the correct helicity from outside. OHTE was built to test this hypothesis and T. Tamano (San Diego) reported that a plasma current of 0.5 MA had been maintained in OHTE for several milliseconds, giving electron temperatures of $0.5 \mathrm{KeV}$, although the desired field configuration may not have been completely established. The $\beta$ values achieved by the RFPs and OHTE are around five per cent and so comparable with those from well-maintained tokamaks, but are obtained at the cost of very high power input, indicating a very short $\tau_{\mathrm{E}}$. Thus, to make their use in power stations more economically attractive, tokamaks will need higher $\beta$ values, whereas the RFP and OHTE devices will need to achieve very much larger values of $\tau_{\mathrm{E}}$.

The spheromak type of configuration has affinity with the RFP but the plasma is formed as a free toroid that generates its own field so that a central conductor through the hole in the plasma doughnut is not required. M. Yamada (Princeton) reported successful generation and control of such plasmas in the S1 spheromak for a period of one millisecond at a plasma current of $0.35 \mathrm{MA}$, but with an electron temperature of only $\sim 50 \mathrm{eV}$. The CTX (Los Alamos) and CCTC-I (Osaka) experiments have also generated such plasmas. Although at an early stage of development, these intriguing devices should tell us a lot about the ability of magnetized plasmas to regulate themselves by automatically relaxing and maintaining a preferred magnetic geometry.

Stellarators are an important class of field configurations which require no plasma current and entirely confine the plasma by the use of externally-generated helical fields. Heliotron $\mathrm{E}$ is stellarator with a highly-sheared magnetic field. It can achieve a $\beta$ value of 2 per cent (K.Uo, Kyoto) and more power may take the value yet higher. Theoretical limits to $\beta$ in stellarators, discussed by B.A. Carreras (Oak Ridge) and J. Johnson (Princeton), are comparable to those for the tokamak. Wendelstein VII A (Garching) is a weak shear $\lambda=2$ stellarator in which, like Heliotron E, the plasma is heated both with neutral beams and with absorption of microwaves at the electron cyclotron resonance. In this machine, $\tau_{\mathrm{E}}$ shows a strong sensitivity to the rotational transform with large dips in $\tau_{\mathrm{E}}$ at rational values (H. Renner, Garching). The weak shear in this case probably allows large regions of the plasma to be interconnected because of the effect of small resonant perturbations. Overall, however, stellarators are now improving on the performance of tokamaks of comparable size, and producing $\tau_{\mathrm{E}}$ values closer to the classical values expected due to collisions alone. Their value of $\tau_{E}$ can therefore be expected to increase as $B^{2}$, a feature not observed in tokamaks; this should be confirmed in the new devices now being constructed at Garching (WVIIAS) and at Oak Ridge (ATF). As stellarators do not require a plasma current they can be operated continuously without plasma disruption. The complex coil system required to establish the helical field is a serious drawback to stellarators but simpler coil designs, which would avoid most of the engineering problems, have been proposed.

The mirror machines, with their essentially linear geometry, are the most studied non-toroidal systems. Many of these devices, some quite large, are in operation. Very ingenious techniques are used to prevent electrons, ions and energy being lost too rapidly along the field lines. In TMX$\mathrm{U}$ (Livermore) and Gamma 10 (Tsukuba), the formation of potential barriers and improved energy confinement have been demonstrated. However the plasma parameters in the centre $\left(N=6 \times 10^{18} \mathrm{~m}^{-3}\right.$, $T=0.2 \mathrm{KeV}$ and $\tau_{\mathrm{E}}=5 \mathrm{~ms}$ ) are still modest compared with toroidal systems.
The heating of plasmas above that provided by the plasma current is technically difficult and has, until recently, relied on injection of fast particle beams. Heating by the absorption of radio-frequency waves has now advanced to the point where MWs of power can be delivered to the plasma. In particular, at the electron cyclotron resonance, radiation with wavelengths of 3.6 and $5 \mathrm{~mm}$ and a power level of $1 \mathrm{MW}$ has been used very effectively both in the Doublet III tokamak (K.H. Burrell) and in the T10 tokamak in Moscow (V.V. Alikaev). The local nature of the power deposition with this form of heating has been used to study energy transport and instability control.

There is no doubt that exciting progress in fusion research has been made in many directions, although extensive development work will be needed for economic power generation from fusion. The next significant step in fusion research is going to be the achievement of ignition and many now believe this can be done.

A.C. Riviere is at the Culham Laboratory, $A b$ ingdon, Oxon $O X 143 D B, U K$.

\title{
New constraint on biomedical research?
}

THE recent prosecution of the Royal College of Surgeons, Parliamentary moves to replace the Cruelty to Animals Act (1876) and the search for in vitro alternatives to animal experimentation may have farreaching effects on research in Britain. A further step in this often acrimonious debate was taken at the inaugural meeting of a new pressure group in Oxford last week.

The conference was opened by the ecologist, Dr J.H.C. Finch. He pointed out that we are, of neccessity, closely linked with all the living organisms with which we share the planet, and that we ignore this at our peril. The wholesale destruction of ecosystems, nuclear proliferation and unethical experimentation were all part of the same anti-life attitude.

Citing electrophysiological evidence that even plants, although they lack a demonstrable nervous system, are capable of responding to painful stimuli, Dr Finch said, "We should respect and revere all the organic world". In the following discussion, several participants pointed out that large and effective organizations already exist for animal rights and suggested the formation of a parallel group to protect plant life. One delegate suggested the term 'chlorophyll based organisms' or CBOs, because they felt the word 'vegetable' can carry derogatory connotations. However, after debate, the name Vegetable Liberation Front was accepted for the new organization.

On the question of agriculture, it was agreed to demand that the government support a major research initiative to develop methods for the de novo molecular synthesis of foodstuffs. Until this was achieved, the spraying of crops with local anaesthetic before to reaping was recommended. No agreement was reached on the ethics of genetic engineering of bacteria for mass production of nutritional protein. One faction insisted that transfection is a violation of genetic integrity, and that this constitutes a subtle form of oppression. "Even bacteria have rights", said one speaker.

There was a strong feeling expressed by many that the movement could only develop, like the Animal Liberation Front, through direct action. Tactics for harvest sabotage, ranging from the immobilization of tractors (but not by stuffing potatoes in their exhaust pipes) to the occupation of grain silos were discussed. Attacks on herbicide factories were rejected because "they could serve a useful role in culling the human predator." The international nature of the struggle was stressed by a fraternal delegate from Holland. He proposed simultaneous demonstrations throughout the EEC outside offices of Interflora, a multinational corporation accused of cruelty to flowers.

Given the urgency of the problems raised, the question of a constitution was deferred until a subsequent meeting. However, a manifesto was drawn up and agreed by the overwhelming majority of delegates. The document was engraved on tablets of stone, since the manufacture of paper was held to entail the suffering of trees, and a copy was posted through windows of the editorial offices of this and other journals.

R.A. Tatouille, University of Brussels. 